\title{
Correlation between osteopontin and caveolin-1 in the pathogenesis and progression of osteoarthritis
}

\author{
TU MIN, LI YU SHENG, CHENG CHAO, TIAN JIAN, GAO SHU GUANG and LEI GUANG HUA \\ Department of Orthopaedics, Xiangya Hospital, Central South University, Changsha, Hunan 410008, P.R. China
}

Received July 6, 2014; Accepted March 17, 2015

DOI: $10.3892 /$ etm.2015.2433

\begin{abstract}
Previous studies have produced contradictory results with regard to the role of osteopontin (OPN) and caveolin-1 in the pathology of osteoarthritis (OA). Thus, the aim of the present study was to investigate the correlation between OPN and caveolin-1 in the pathogenesis and progression of OA. Cartilage tissue samples were obtained from 50 individuals, of which 40 had been diagnosed with OA and 10 were normal healthy individuals. The samples were ascribed to four groups, namely the normal, minor, moderate and severe groups, on the basis of the improved Mankin grading system. Immunohistochemistry was applied to analyse the expression of OPN and caveolin-1. OPN and caveolin-1 were detected in the tissues of all four groups. The mutual comparisons of OPN expression levels among the groups revealed statistically significant differences $(\mathrm{P}<0.05)$. In addition, the mutual comparisons of caveolin-1 expression levels among the four groups demonstrated statistically significant differences $(\mathrm{P}<0.05)$, with the exception of that between the moderate and severe groups $(\mathrm{P}>0.05)$. Improved Mankin grading system scores were shown to correlate with the average grey level of OPN expression in each group $(\mathrm{r}=-0.824, \mathrm{P}<0.01)$ and the average grey level of caveolin-1 expression $(\mathrm{r}=0.725, \mathrm{P}<0.01)$. Furthermore, a statistically significant negative correlation was observed between the average grey levels of OPN and caveolin-1 expression $(\mathrm{r}=-0.676, \mathrm{P}<0.05)$. Therefore, the results of the present study indicated that the correlation between OPN and caveolin-1 may play a significant role in the pathogenesis and progression of $\mathrm{OA}$.
\end{abstract}

Correspondence to: Dr Lei Guang Hua, Department of Orthopaedics, Xiangya Hospital, Central South University, 87 Xiangya Road, Changsha, Hunan 410008, P.R. China

E-mail: wuqinzhe1981811@gmail.com

Abbreviations: OA, osteoarthritis; OPN, osteopontin; ECM, extracellular matrix; PBS, phosphate-buffered saline; MIAS, medical image analysis software

Key words: osteopontin, caveolin-1, osteoarthritis, Mankin classification

\section{Introduction}

Osteoarthritis (OA) is a highly prevalent joint disease, which is mainly characterised by the degradation of articular cartilage, thickening of subchondral bone, synovial inflammation and the formation of osteophytes (1). Data from the World Health Organisation ascertains that worldwide, $\sim 10 \%$ of men and $18 \%$ of women, as well as $\sim 65 \%$ of all those aged $>60$ years, have symptomatic OA (2). Despite the high prevalence of OA, the aetiology and pathology of OA have not yet been fully elucidated. The development and progression of OA are mediated by multiple factors, including genetics, epigenetics, biochemistry and mechanics (3-5). Several lines of research have demonstrated that a disturbance in the balance of anabolic and catabolic factors, which maintain the homeostasis of cartilage, plays a vital role in OA (6). Understanding the specific role of each factor is important for the treatment of $\mathrm{OA}$, and is also vital for creating effective therapeutic interventions targeting the development and progression of OA. Since numerous molecular events occur in OA chondrocytes during hyaline cartilage degeneration, several studies have been conducted to investigate the role of osteopontin (OPN) and caveolin-1 in OA $(7,8)$.

OPN is an acidic phosphorylated matrix protein secreted by numerous cell types, such as osteoclasts, macrophages, lymphocytes and epithelial cells (9). OPN is present in the extracellular matrix (ECM) of mineralised tissues and the extracellular fluids at sites of inflammation (10). Previous studies have demonstrated that OPN plays a significant role in cell adhesion, migration and metastasis $(11,12)$. In the last decade, the vast majority of research has indicated that the mRNA expression levels of OPN in OA cartilage and synovium fluid are clearly higher compared with normal cartilage, and that the expression levels of OPN protein and OPN mRNA are positively associated with the severity of the disease (13-15). By contrast, a previous study reported that an OPN deficiency resulted in aging-associated and instability-induced $\mathrm{OA}$ in OPN-deficient mice, suggesting that OPN may defer the pathogenesis in OA (16). These observations strongly indicate that OPN has complex roles in joint homeostasis and in the pathogenesis of OA by modulating multiple targets of cells in the joint; however, the role of OPN in OA remains poorly understood.

Caveolin-1, the major structural component of caveolae, is a small flask-shaped plasma membrane invagination $(17,18)$. 
The protein has been proposed to function as a scaffolding protein, mediating numerous physiological and pathological processes, including caveolae biogenesis, vesicular transport, cholesterol homeostasis, signal transduction and tumourigenesis $(19,20)$. Previously, the expression of caveolin-1 was analysed in normal and OA human knee joint cartilage (21). Caveolin-1 has been reported to play a role in the pathogenesis of OA through the involvement of caveolin-1-induced downregulation of articular chondrocytes (22).

Previous observations have revealed that caveolin-1 is associated with $\beta_{1}$-integrin in human chondrocytes, indicating that caveolin-1 and $\beta_{1}$-integrin are part of the same complex (21). $\beta_{1}$-integrin plays a direct role in the adhesion between the cell and the ECM, in which OPN is involved through binding to the OPN receptor (23). Since $\beta_{1}$-integrin mediates the role of OPN and colocalises with caveolin-1, the expression of caveolin-1 was hypothesised to correlate with OPN expression through the signalling pathway in which $\beta_{1}$-integrin mediates OPN. Therefore, to investigate the hypothesis, the expression levels of OPN and caveolin-1 in different degrees of impaired cartilage samples from human subjects with OA were determined, and the correlation between OPN and caveolin-1 was analysed. The aim of the present study was to provide a more comprehensive understanding of OPN and caveolin-1 in OA.

\section{Materials and methods}

Patients and cartilage samples. A total of 40 patients (age, 52-71 years) with primary knee OA and 10 normal healthy individuals were enrolled in the study. Patients with OA were considered eligible if the clinical and radiological data met the criteria of the American College of Rheumatology (24) and were carefully reviewed to exclude any forms of secondary OA or other inflammatory joint diseases, including rheumatoid arthritis and any other types of arthritis. All patients provided informed consent, and this study was approved by the ethics committee of Xiangya Hospital, Central South University (Changsha, China). In total, 40 osteoarthritic cartilage samples were collected from the 40 patients with primary OA undergoing a total knee arthroplasty. The normal samples were obtained from the knees of 10 post-mortem donors (age, 32-41 years), with no history of joint pain. Full ethical consent was obtained from all the donors and families. Osteoarthritic changes were classified histomorphologically using the Mankin grading system (25) as follows: Mankin score 0, normal cartilage with a smooth surface and a regular zonal distribution of chondrocytes; Mankin score 1-4, cartilage surface shows fibrillations and a superficial loss of proteoglycans (safranin-O staining), but the zonal structure is intact; Mankin score 5-8, cartilage samples have clefts reaching down to the middle cartilage zone, and clusters of proliferating chondrocytes are present; Mankin score $\geq 9$, severely affected cartilage samples with clefts reaching down to the deep zone, in which the tangential zone is lost and chondrocyte clusters are present. Of the patients included in the study, 9 samples were classified as Mankin score 0,10 samples were determined to be Mankin score 1-4, 15 samples were classified as Mankin score 5-8 and 13 samples were classified with a Mankin score of $\geq 9$. Biopsies (cartilage/bone samples) were obtained from the lateral and medial sides of the tibia plateau, including the loading zone.
Cartilage/bone samples $(1.0 \mathrm{~cm}$ thick) with a cartilage surface of $2.0 \times 0.5 \mathrm{~cm}$ were incubated in freshly prepared $4 \%(\mathrm{w} / \mathrm{v})$ paraformaldehyde, buffered with $0.01 \mathrm{M}$ sodium phosphate (pH 7.4) containing $0.14 \mathrm{M} \mathrm{NaCl}$ (phosphate-buffered saline; PBS), for $12 \mathrm{~h}$ at $4^{\circ} \mathrm{C}$. The tissue samples were decalcified in diethylpyrocarbonate-treated $0.2 \mathrm{M}$ EDTA $(\mathrm{pH}$ 8.0) for 8 weeks at $4^{\circ} \mathrm{C}$. The buffer was changed twice a week. The tissue specimens were subsequently dehydrated in a grading concentration of ethanol and xylene, and finally embedded in paraffin.

Immunohistochemistry. OPN protein and caveolin-1 expression levels were examined by immunohistochemistry using an OPN antibody (ab8488; Abcam, Cambridge, UK) and caveolin-1 antibody (clone N-20; Santa Cruz Biotechnology, Inc., Wembley, UK), respectively, according to the manufacturer's instructions. Following deparaffinisation with xylene and rehydration through a series of graded ethanol solutions, the sections were treated with $3 \%$ hydrogen peroxide for $10 \mathrm{~min}$ and then microwaved in $10 \mathrm{mM}$ citrate buffer ( $\mathrm{pH}$ 6.0) to unmask the epitopes. Subsequently, the sections were incubated with the OPN antibody at a dilution of 1:150 for $1 \mathrm{~h}$. After washing, a horseradish peroxidase/Fab polymer conjugate (PicTure ${ }^{\mathrm{TM}}$-Plus kit; Zymed Life Technologies, Carlsbad, CA, USA) was applied to the sections for $30 \mathrm{~min}$. Finally, the sections were incubated with diaminobenzidine for $5 \mathrm{~min}$ to develop the signals. A negative control was simultaneously prepared by omitting the primary antibody. The sections were assessed by a pathologist who was blind to the clinical data. To evaluate the expression of OPN, the sections were examined under an Olympus microscope (magnification, x100; Olympus Corporation, Tokyo, Japan). Positive OPN immunostaining was defined as detectable immunoreactivity in the perinuclear or other cytoplasmic regions in the chondrocytes. The relative OPN distribution in the cartilage tissue was visualised and quantified as average grey values. Semi-quantitative assessment of the mean average grey values for OPN expression was performed on scanned autoradiograms using medical image analysis software (MIAS)-4400 and Image J software. Grey-scale images were captured and converted to absorbance units, and a region from the cartilage surface to the cartilage-bone junction was analysed. Densities were normalised against those with PBS, and the experiment was repeated three times. To reduce the error arising from the small variation in section thickness, a total of three sections per sample were measured and averaged. Therefore, the final data, applied in all analyses, consisted of the mean value of three independent measurements representing the average levels of OPN in the articular cartilage. The coefficient of variation of OPN expression in the articular cartilage was $<2 \%$. The aforementioned procedure was conducted in the same manner to assess caveolin-1 expression.

Statistical analysis. Static grey analysis from the MIAS was applied to measure the average grey values in 10 randomly selected regions of the OPN and caveolin-1 immunohistochemical slices. SPSS software for Windows (version 19.0; IBM SPSS, Armonk, NY, USA) was used for data management and statistical analysis. One way analysis of variance was employed to examine the differences in the mean values 

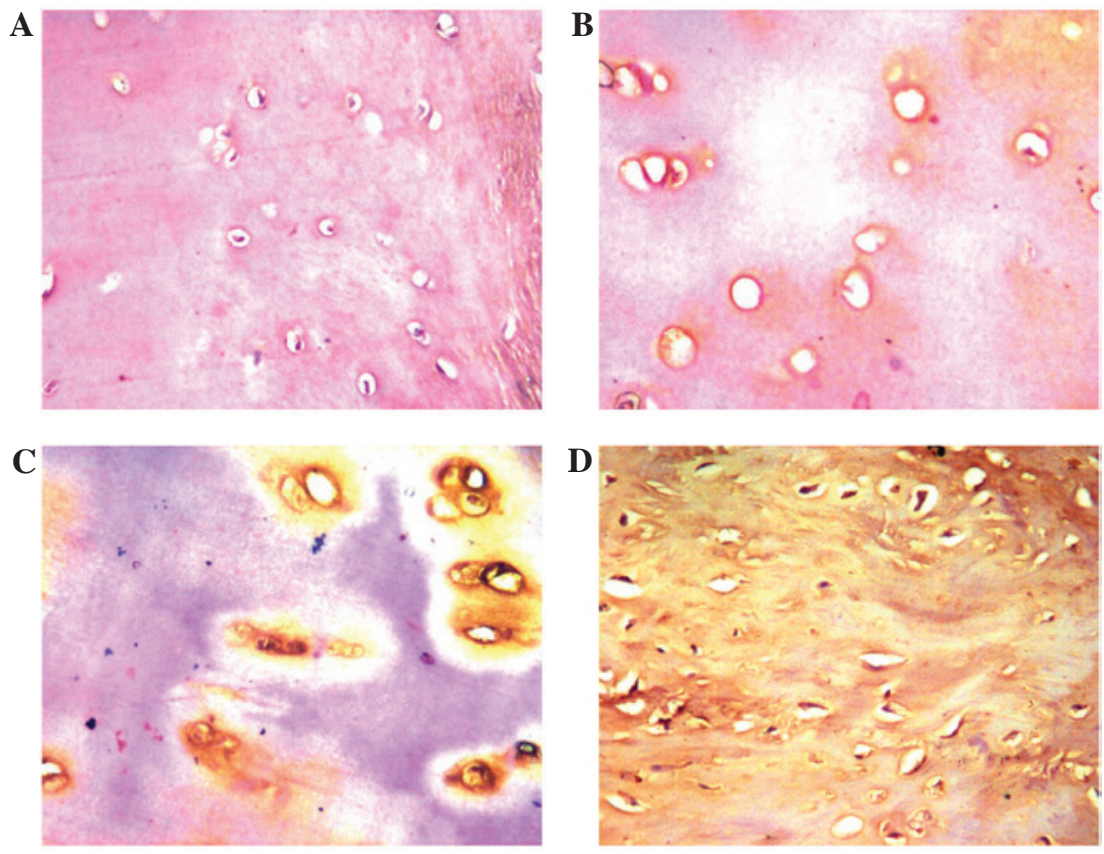

Figure 1. Expression of osteopontin was detected in the (A) normal, (B) minor, (C) moderate and (D) severe groups of cartilage degradation using immunohistochemistry (magnification, x100).

between multiple groups. In addition, Spearman's correlation and linear regression were conducted to determine the correlations between the average grey values for OPN and caveolin-1 in the articular cartilage and the Mankin score of OA. Results are presented as the mean \pm standard error of the mean. $\mathrm{P}<0.05$ was considered to indicate a statistically significant difference.

\section{Results}

Mankin scoring system in each group. A total of 50 biopsies, which were obtained from 40 patients and 10 normal individuals, were ascribed respectively to four groups (normal, minor, moderate and severe), according to the Mankin grading system. Three biopsies were excluded due to unsuccessful cutting from the cartilage and a failure in the procedure of immunohistochemistry. Ultimately, 47 samples were analysed. Grouping of the cartilage tissue samples according to the Mankin scoring system are shown in Table I.

Enhanced expression of OPN protein in OA cartilage tissues. OPN expression levels in the various degrees of impaired cartilage tissues were compared, as shown in Fig. 1. OPN, which is located in the ECM, was shown to be expressed in the normal and OA groups. The severe group exhibited a higher OPN expression level compared with the moderate, minor and normal groups (average grey value, 97.98 \pm 12.46 vs. $114.14 \pm 10.85,125.33 \pm 8.02$ and $140.06 \pm 12.17$, respectively; Table II). A low grey value indicates higher expression levels. The mutual comparisons between these groups revealed statistically significant differences $(\mathrm{P}<0.05)$. Thus, the results indicated that expression of the OPN protein was strongly increased in the impaired cartilage.

Decreased expression of caveolin-1 protein in OA cartilage tissues. To investigate the function of caveolin-1 in OA, the
Table I. Mankin scoring system of cartilage in each group.

\begin{tabular}{lcr}
\hline Group & Sample (n) & Mankin score \\
\hline Normal & 9 & $0.55 \pm 0.53$ \\
Minor & 10 & $3.90 \pm 1.12$ \\
Moderate & 15 & $7.77 \pm 1.09$ \\
Severe & 13 & $11.93 \pm 1.44$ \\
\hline
\end{tabular}

Results are presented as the mean \pm standard error of the mean.

expression levels in the various degrees of impaired cartilage tissues were compared (Fig. 2). Caveolin-1 was shown to be expressed in the normal and OA groups. However, the severe group exhibited lower caveolin-1 expression levels compared with the moderate, minor and normal groups (average grey value, $140.10 \pm 15.29$ vs. $131.72 \pm 19.32,112.00 \pm 23.73$ and $85.90 \pm 15.11$, respectively; Table II). The mutual comparisons between these groups were demonstrated to be statistically significant $(\mathrm{P}<0.05)$, with the exception of that between the moderate and severe groups $(\mathrm{P}>0.05)$.

Correlations between OPN, caveolin-1 and Mankin scoring. To investigate the correlation between OPN and caveolin-1 in OA, SPSS software was applied to assess the average grey value for each protein and the Mankin scoring. Correlations between the average grey value of OPN and caveolin-1 with the Mankin scoring are shown in Fig. 3. For the correlation between the average grey value of OPN and the Mankin scoring, Pearson's correlation coefficient was determined to be $\mathrm{r}=-0.824(\mathrm{P}<0.01)$. In addition, Pearson's correlation coefficient for the correlation between the average grey value of caveolin-1 and the Mankin scoring was $\mathrm{r}=0.725(\mathrm{P}<0.01)$. 
Table II. Average grey value levels of OPN and caveolin-1 in each group.

\begin{tabular}{lccc}
\hline Group & Sample (n) & OPN value & Caveolin-1 value \\
\hline Normal & 9 & $140.06 \pm 12.17$ & $85.90 \pm 15.11$ \\
Minor & 10 & $125.33 \pm 8.02^{\mathrm{a}}$ & $112.00 \pm 23.73^{\mathrm{a}}$ \\
Moderate & 15 & $114.14 \pm 10.85^{\mathrm{a}, \mathrm{b}}$ & $131.72 \pm 19.32^{\mathrm{a}}$ \\
Severe & 13 & $97.98 \pm 12.46^{\mathrm{a}, \mathrm{b}, \mathrm{c}}$ & $140.10 \pm 15.29^{\mathrm{a}, \mathrm{b}, \mathrm{c}}$
\end{tabular}

${ }^{\mathrm{a}} \mathrm{P}<0.01$, vs. normal group; ${ }^{\mathrm{b}} \mathrm{P}<0.01$, vs. minor group; ${ }^{\mathrm{c}} \mathrm{P}>0.05$, vs. moderate group. OPN, osteopontin.
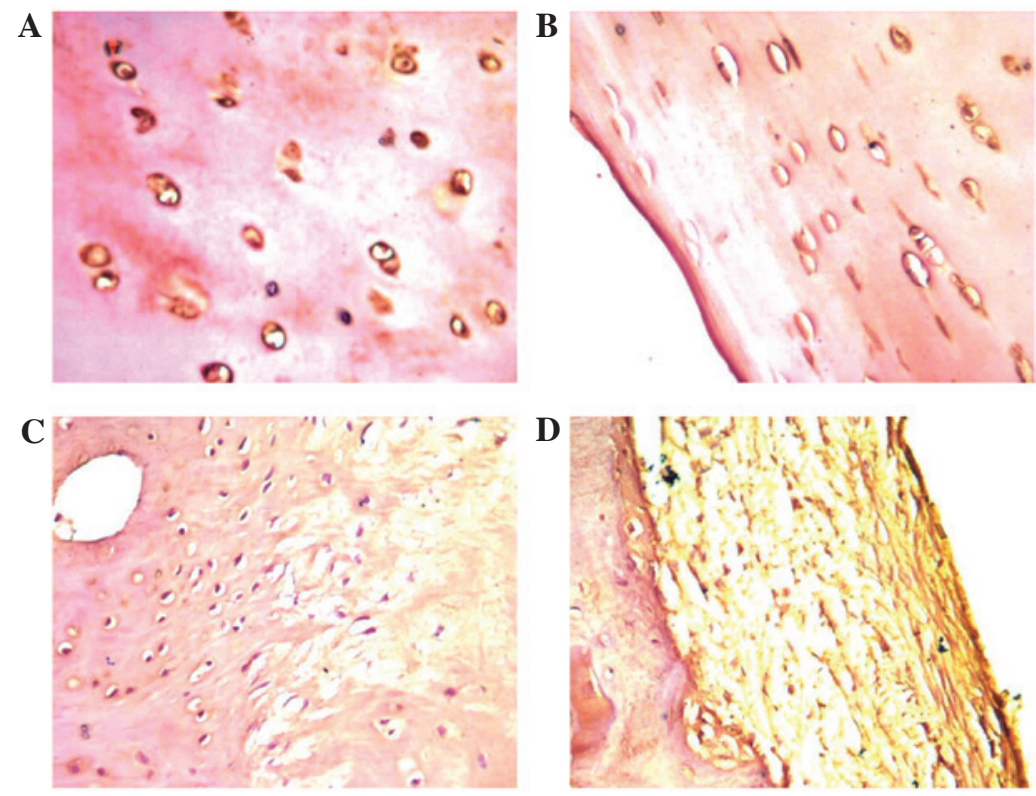

Figure 2. Caveolin-1 expression was detectable in the (A) normal, (B) minor, (C) moderate and (D) severe groups of cartilage degradation using immunohistochemistry (magnification, $\mathrm{x} 100$ ).
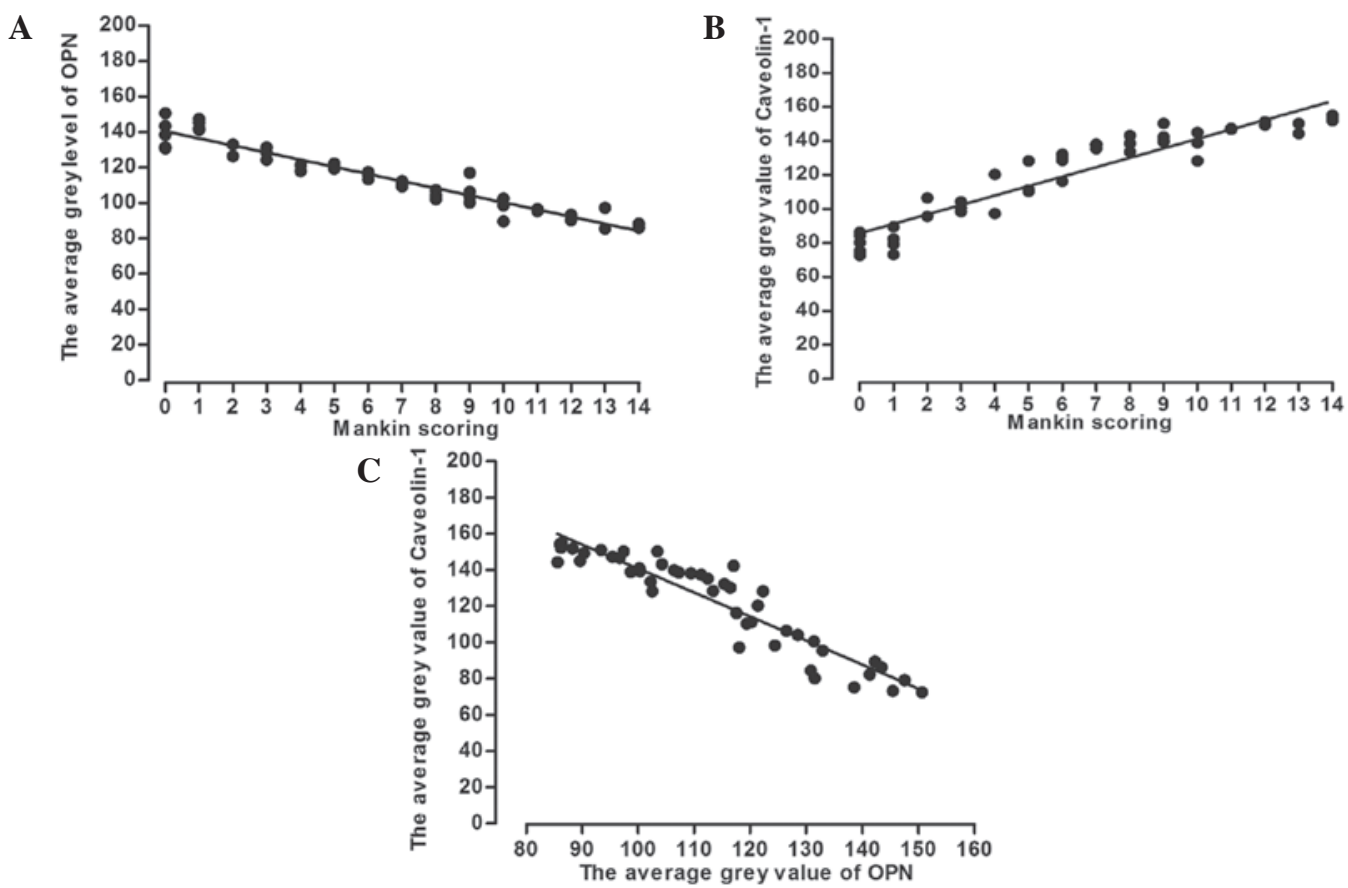

Figure 3. (A) Correlation between the average grey value of OPN and Mankin scoring. Pearson's correlation coefficient, $r=-0.824$ (P<0.01). (B) Correlation between the average grey value of caveolin-1 and Mankin scoring. Pearson's correlation coefficient, $r=0.725(\mathrm{P}<0.01)$. (C) Correlation between the average grey values of OPN and caveolin-1. Pearson's correlation coefficient, $r=-0.676(\mathrm{P}<0.01)$. OPN, osteopontin. 
Furthermore, the correlation between the average grey values of OPN and caveolin-1 was determined to have a Pearson's correlation coefficient of $\mathrm{r}=-0.676(\mathrm{P}<0.01)$.

\section{Discussion}

As an ECM protein, OPN plays a significant role in the process of cell adhesion, migration and metastasis (9). OPN is expressed by various cells and tissues, such as the kidney, placenta, neoplastic cells, lining epithelial cells, islet cells and activated T cells; however, the main source of OPN is bone cells, including chondrocytes and osteoclasts. The present study demonstrated that OPN plays a crucial role in the progression of mineralisation and absorption of bone matrix. A previous study reported that OPN may function as an inhibitor involved in the molecular pathogenesis of OA, contributing to the progressive degeneration of articular cartilage (26), whereas an additional study drew the opposite conclusion (27). However, these two studies did not investigate OPN overexpression. In the present study, OPN expression was observed in the normal and OA groups. However, the severe OA group were shown to have higher OPN expression levels compared with the moderate, minor and normal groups. Based on this observation, a statistically significant difference was observed in the expression of OPN between OA and normal cartilage, and the articular cartilage OPN expression was shown to correlate with the severity level of cartilage dysfunction. Thus, the results indicated that OPN may play a role in the pathology and progression of OA as a destructive factor.

In addition to participating in the formation of caveolae, caveolin-1 is known to directly interact with multiple signalling proteins via its scaffolding domain in order to regulate their activity. These proteins include important regulators of cell transformation and growth (28). Previously, caveolin-1 expression was demonstrated in normal human knee joint cartilage by immunohistochemical analysis (21). The current findings also detected caveolin-1 expression in the normal cartilage, as well as in the different pathological levels of OA cartilage, with a lower expression of caveolin-1 being accompanied by a higher regression of cartilage. Therefore, the results of the present study demonstrated that caveolin-1 may be involved in the degradation of articular cartilage in OA, which differs from previous results that hypothesised that caveolin-1 played a role in the pathogenesis of OA through the promotion of chondrocyte downregulation. Further research is required in order to elucidate the exact role of caveolin-1 in OA.

Signal transduction is the major function of caveolin-1. Previous studies have demonstrated that caveolin-1 and $\beta_{1}$-integrin are part of the same complex in human chondrocytes $(29,30)$. Integrin, which is one of the transmembrane glycoproteins, is located throughout the cell membrane. Integrins interact with a variety of cellular adhesive molecules and are regarded as a family of adhesion molecules. As the main receptor of the ECM, integrin induces the adhesion process of the cell. Schwab et al (21) detected the colocalisation between caveolin- 1 and $\beta_{1}$-integrin using double staining methods and immune electron microscopy in human samples of knee articular cartilage, and drew the conclusion that $\beta_{1}$-integrin had a close association with the three subtypes of caveolin, which may be different parts of the same complex. Based on these observations, all the caveolin subtypes were hypothesised to be indirectly involved in processes of the cell and ECM through participating in the signalling pathway induced by $\beta_{1}$-integrins in chondrocytes. OPN induces adhesion between the cell and ECM by binding to the OPN receptor, and $\beta_{1}$-integrin may directly play a role in this process.

In the present study, a correlation was observed between cartilage OPN expression and the severity level of cartilage dysfunction; however, caveolin-1 expression was shown to exhibit the opposite trend. In addition, the correlation between OPN and caveolin-1 expression was statistically significant, indicating that caveolin-1 may correlate with OPN through the signalling pathway in which $\beta_{1}$-integrin mediates OPN.

However, the present study had several limitations. Firstly, the sample size was not sufficiently large to arrive at any definitive conclusions. Secondly, the true pathogenesis of OA may not be fully reflected when representing the progression of OA with the articular cartilage degeneration degree.

In conclusion, the correlation between OPN and caveolin-1 may play a substantial role in the pathogenesis and progression of OA. However, further research is required to elucidate the contribution of OPN and caveolin-1 to the pathogenesis of the degenerative process of $\mathrm{OA}$.

\section{Acknowledgements}

This study was supported by grants from the National Natural Science Foundation of China (no. 81272034) and the Fundamental Research Funds for the Central Universities of Central South University (2013 zzts081).

\section{References}

1. Poole AR: An introduction to the pathophysiology of osteoarthritis. Front Biosci 4: D662-D670, 1999.

2. Woolf AD and Pfleger B: Burden of major musculoskeletal conditions. Bull World Health Organ 81: 646-656, 2003.

3. Loughlin J: Genetics of osteoarthritis and potential for drug development. Curr Opin Pharmacol 3: 295-299, 2003.

4. Im GI and Choi YJ: Epigenetics in osteoarthritis and its implication for future therapeutics. Expert Opin Biol Ther 13: 713-721, 2013.

5. Murray RC, Zhu CF, Goodship AE, Lakhani KH, Agrawal CM and Athanasiou KA: Exercise affects the mechanical properties and histological appearance of equine articular cartilage. Journal of orthopaedic research. J Orthop Res 17: 725-731, 1999.

6. Aigner T, Soeder S and Haag J: Il-1 beta and BMPS-interactive players of cartilage matrix degradation and regeneration. Eur Cell Mater 12: 49-56, 2006.

7. Dai SM, Shan ZZ, Nakamura H, et al: Catabolic stress induces features of chondrocyte senescence through overexpression of caveolin 1: Possible involvement of caveolin 1-induced down-regulation of articular chondrocytes in the pathogenesis of osteoarthritis. Arthritis Rheum 54: 818-831, 2006.

8. Gao SG, Cheng L, Zeng C, et al: Usefulness of specific OA biomarkers, thrombin-cleaved osteopontin, in the posterior cruciate ligament OA rabbit model. Osteoarthritis Cartilage 21: 144-150, 2013

9. Denhardt DT and Guo X: Osteopontin: a protein with diverse functions. FASEB J 7: 1475-1482, 1993.

10. Sodek J, Chen J, Nagata T, et al: Regulation of osteopontin expression in osteoblasts. Ann N Y Acad Sci 760: 223-241, 1995.

11. Rangaswami H, Bulbule A and Kundu GC: Osteopontin: role in cell signaling and cancer progression. Trends Cell Biol 16: 79-87, 2006.

12. Rittling SR and Chambers AF: Role of osteopontin in tumour progression. Br J Cancer 90: 1877-1881, 2004. 
13. Gao SG, Li KH, Zeng KB, Tu M, Xu M and Lei GH: Elevated osteopontin level of synovial fluid and articular cartilage is associated with disease severity in knee osteoarthritis patients. Osteoarthritis Cartilage 18: 82-87, 2010.

14. Hasegawa M, Segawa T, Maeda M, Yoshida T and Sudo A: Thrombin-cleaved osteopontin levels in synovial fluid correlate with disease severity of knee osteoarthritis. J Rheumatol 38: 129-134, 2011.

15. Honsawek S, Tanavalee A, Sakdinakiattikoon M, Chayanupatkul $\mathrm{M}$ and Yuktanandana P: Correlation of plasma and synovial fluid osteopontin with disease severity in knee osteoarthritis. Clin Biochem 42: 808-812, 2009.

16. Matsui Y, Iwasaki N, Kon S, et al: Accelerated development of aging-associated and instability-induced osteoarthritis in osteopontin-deficient mice. Arthritis Rheum 60: 2362-2371, 2009.

17. Lisanti MP, Scherer PE, Tang Z and Sargiacomo M: Caveolae, caveolin and caveolin-rich membrane domains: a signalling hypothesis. Trends Cell Biol 4: 231-235, 1994.

18. Liu P, Rudick M and Anderson RG: Multiple functions of caveolin-1. J Biol Chem 277: 41295-41298, 2002.

19. Engelman JA, Chu C, Lin A, et al: Caveolin-mediated regulation of signaling along the p42/44 MAP kinase cascade in vivo. A role for the caveolin-scaffolding domain. FEBS Lett 428: 205-211, 1998

20. Okamoto T, Schlegel A, Scherer PE and Lisanti MP: Caveolins, a family of scaffolding proteins for organizing 'preassembled signaling complexes' at the plasma membrane. J Biol Chem 273: 5419-5422, 1998

21. Schwab W, Kasper M, Gavlik JM, Schulze E, Funk RH and Shakibaei M: Characterization of caveolins from human knee joint cartilage: expression of caveolin-1, -2 and -3 in chondrocytes and association with integrin beta1. Histochem Cell Biol 113: $221-225,2000$
22. Dai SM, Shan ZZ, Nakamura H, et al: Catabolic stress induces features of chondrocyte senescence through overexpression of caveolin 1: possible involvement of caveolin 1-induced down-regulation of articular chondrocytes in the pathogenesis of osteoarthritis. Arthritis Rheum 54: 818-831, 2006.

23. Ross FP, Chappel J, Alvarez JI, et al: Interactions between the bone matrix proteins osteopontin and bone sialoprotein and the osteoclast integrin alpha $\mathrm{v}$ beta 3 potentiate bone resorption. J Biol Chem 268: 9901-9907, 1993.

24. Aletaha D, Neogi T, Silman AJ, et al: 2010 rheumatoid arthritis classification criteria: An American College of Rheumatology/European League Against Rheumatism collaborative initiative. Ann Rheum Dis 69: 1580-1588, 2010.

25. van der Sluijs JA, Geesink RG, van der Linden AJ, Bulstra SK, Kuyer R and Drukker J: The reliability of the Mankin score for osteoarthritis. J Orthop Res 10: 58-61, 1992.

26. Attur MG, Dave MN, Stuchin S, et al: Osteopontin: an intrinsic inhibitor of inflammation in cartilage. Arthritis Rheum 44: 578-584, 2001

27. Matsui Y, Iwasaki N, Kon S, et al: Accelerated development of aging-associated and instability-induced osteoarthritis in osteopontin-deficient mice. Arthritis Rheum 60: 2362-2371, 2009

28. van Golen KL: Is caveolin-1 a viable therapeutic target to reduce cancer metastasis? Expert Opin Ther Targets 10: 709-721, 2006.

29. Jokhadar SZ, Majhenc J, Svetina S and Batista U: Positioning of integrin $\beta 1$, caveolin-1 and focal adhesion kinase on the adhered membrane of spreading cells. Cell Biol Int 37: 1276-1284, 2013.

30. Radel C, Carlile-Klusacek M and Rizzo V: Participation of caveolae in betal integrin-mediated mechanotransduction. Biochem Biophys Res Commun 358: 626-631, 2007. 